NOTICE: this is the author's version of a work that was accepted for publication in International Journal of Machine Tools and Manufacture. Changes resulting from the publishing process, such as peer review, editing, corrections, structural formatting, and other quality control mechanisms may not be reflected in this document. Changes may have been made to this work since it was submitted for publication. A definitive version was subsequently published in International Journal of Machine Tools and Manufacture, Volume 46, Issue 14, November 2006, http://dx.doi.org/10.1016/j.ijmachtools.2005.11.012 


\title{
Prediction of cutting forces in machining of metal matrix composites
}

\author{
A. Pramanik, L. C. Zhang* and J. A. Arsecularatne \\ School of Aerospace, Mechanical and Mechatronic Engineering, The University of Sydney, Sydney, \\ NSW 2006, Australia,
}

\begin{abstract}
This paper presents a mechanics model for predicting the forces of cutting aluminium-based $\mathrm{SiC} / \mathrm{Al}_{2} \mathrm{O}_{3}$ particle reinforced MMCs. The force generation mechanism was considered to be due to three factors: (a) the chip formation force, (b) the ploughing force, and (c) the particle fracture force. The chip formation force was obtained by using Merchant's analysis but those due to matrix ploughing deformation and particle fracture were formulated, respectively, with the aid of the slip line field theory of plasticity and the Griffith theory of fracture. A comparison of the model predictions with the authors' experimental results and those published in the literature showed that the theoretical model developed has captured the major material removal/deformation mechanisms in MMCs and describes very well the experimental measurements.
\end{abstract}

Keywords: machining; metal matrix composites; force modeling; PCD tool

\section{Nomenclature}

A Cross-sectional area of groove $\left(\mathrm{mm}^{2}\right)$

$A_{c} \quad$ Cross-sectional area of the cut $\left(\mathrm{mm}^{2}\right)$

$B, C \quad$ Constants in equation (21)

$a \quad$ Depth of cut (mm)

$d_{p} \quad$ Average diameter of particles (mm)

$F_{c} \quad$ Total force in the cutting direction $(\mathrm{N})$

$E_{f t} \quad$ Total energy required for particle fracture (J)

$F_{c c} \quad$ Cutting force for chip formation (N)

$F_{c f} \quad$ Cutting force for particle fracture $(\mathrm{N})$

\footnotetext{
* Corresponding author (zhang@aeromech.usyd.edu.au)
} 
$F_{c p} \quad$ Cutting force for ploughing (N)

$F_{f} \quad$ Total force for particle fracture (N)

$F_{t} \quad$ Total force in thrust direction (N)

$F_{t c} \quad$ Thrust force for chip formation (N)

$F_{t f} \quad$ Thrust force for particle fracture (N)

$F_{t p} \quad$ Thrust force for ploughing (N)

$f \quad$ Feed $(\mathrm{mm} / \mathrm{rev})$

$h \quad$ Depth of groove $(\mu \mathrm{m})$

$H \quad$ Height of ploughing zone $(\mu \mathrm{m})$

L Total length of cutting (m)

$L_{e i} \quad$ Effective cutting edge length of indenter (mm)

l $\quad$ Active cutting edge length (mm)

$r_{c} \quad$ Chip thickness ratio

$r_{\varepsilon} \quad$ Tool nose radius $(\mathrm{mm})$

$r_{n} \quad$ Cutting edge radius $(\mathrm{mm})$

$t \quad$ Cut thickness (mm)

$v \quad$ Cutting speed $(\mathrm{m} / \mathrm{min})$

w $\quad$ Width of cut (mm)

$\beta \quad$ Mean friction angle (degree)

$\gamma \quad$ Rake angle (degree)

$\kappa_{r} \quad$ Approach angle (degree)

$\kappa_{r}^{*} \quad$ Approach angle for equivalent cutting edge (degree)

$\delta \quad$ Direction of resultant particle fracture force (Fig.2)

$\mu_{f} \quad$ Specific energy for particle fracture due to scratching $\left(\mathrm{J} / \mathrm{mm}^{3}\right)$

$\mu_{g} \quad$ Particle fracture energy per unit cutting edge length $(\mathrm{J} / \mathrm{mm})$

$\tau_{s} \quad$ Shear strength of MMC (MPa)

$\tau_{s m} \quad$ Shear strength of matrix material (MPa) 
$\phi \quad$ Shear angle (degree)

\section{Introduction}

Composite materials are a result of the continuous attempts to develop new engineering materials with low weight to strength ratios and improved properties. Among modern composites materials, ceramic fiber/particulate/whisker reinforced metal matrix composites (MMCs) are finding increased applications due to their favorable mechanical properties [1] such as improved strength, stiffness and increased wear resistance over unreinforced alloys [2]. Since 1970s, MMCs have been successfully applied in the aeronautic and aerospace industries [3] and nowadays their use is gaining importance. As these composites contain very high hardness strengthening particles, the cutting tool tends to wear severely resulting in difficulties in machining [4]. Thus, in manufacturing, difficulties associated with precision and highly efficient machining of composite materials have become an important issue. According to reference [5], initial research on cutting and grinding of MMCs was started in 1985 and many research papers on machining of aluminium matrix composites have since been published. Almost all the investigations on cutting aluminium matrix composites can be divided into three categories as follows.

(1) Experimental studies that compare different tools and/or coatings for machining MMCs [6-8].

(2) Experimental studies on the performance of PCD tools, machined surface, and optimization of cutting parameters, tool geometry and work piece compositions [1, 4, 9-22].

(3) Empirical and numerical studies related to tool life [1, 13, 23].

It was noted that except for an energy based model [24] to predict the cutting force in orthogonal machining of MMCs, no other work on modeling has been reported. In [24], the estimated energy (based on the deformation in the primary and secondary zones and reinforcement particle displacement and fracture) was used to determine only the cutting force. This model was based on assumptions that the energy in the secondary deformation zone was one third of that of primary deformation zone and that the initial and final crack lengths of ceramic particles were $1 \mu \mathrm{m}$ and the circumference of a particle respectively. However, the first assumption was based on the results obtained for (monolithic) steel work 
materials while no information was given to justify the second assumption. In addition, energy due to ploughing was not considered.

It seems that there has been considerable research in machining of MMCs but most of it is experimental and hence the development of an analytical machining model for MMC is essential. The present study aims at developing an analytical mechanics model, based on the major material removal mechanisms, to predict the forces in cutting aluminium based MMCs reinforced by ceramic particles.

\section{Modeling}

The mechanism of machining MMCs is complex since the composites contain hard ceramic particles. The strength and properties of MMCs, and hence their machining forces, depend on the matrix, the reinforcement and their interface [4].

The machining tests on aluminium alloy based MMCs (reinforced with 20 vol\% SiC) carried out by Lin et al [25] and El-Gallab et al [26] indicated that, with sharp PCD tools, at depths of cut up to $2.5 \mathrm{~mm}$, feeds up to $0.45 \mathrm{~mm} / \mathrm{rev}$ and speed in the range $300-700 \mathrm{~m} / \mathrm{min}$, continuous chips were formed. Under similar conditions with worn tools or at higher depths/feeds, semi-continuous chips were observed. Similar observations on the type of formed chip were also made by Karthikeyan et al [27] who machined MMCs (reinforced with 10-25 vol\% SiC) using tungsten carbide tools at speeds of $50-150 \mathrm{~m} / \mathrm{min}$.

Thus it can be seen that the type of formed chip during machining MMCs changes with the cutting conditions and/or conditions of the tool. Although cracks have been observed at the root of the chip, there are also similarities in the chip formation mechanism of MMCs to that of monolithic materials [28]. For example, the flow lines formed [26, 29] with particles in the MMCs are similar to those due to deformation of grain boundaries, etched patterns in steel, aluminium, titanium or brass [28]. In the chip root region, these ceramic particles have been observed to align along the shear plane [29]. On the other hand, the existence of fractured and displaced particles on the machined surface [24, 26, 29] indicates that particle fracture and displacement also occurs during machining of MMC. Moreover, in an investigation of single point scratching of four different aluminium alloy based MMCs (reinforced with $\mathrm{SiC} / \mathrm{Al}_{2} \mathrm{O}_{3}$ particles), Yan and Zhang [30] found that scratching process was composed of rubbing, 
ploughing, plastic cutting and particle fracture. They were able to determine the energy due to particle fracture by subtracting the energies due to ploughing, plastic cutting and rubbing from total energy of scratching.

The above discussion indicates that, chip formation, ploughing, and particle fracture and displacement can be considered as the major contributions to the force of machining a MMC.

A typical orthogonal cutting process of an MMC is shown in Fig.1. Chip formation is assumed to be due to shearing that occurs at the shear plane $A B$ and is considered to occur only in this region. This process is similar to normal orthogonal cutting of a monolithic material with a sharp tool.

The process of ploughing occurs due to material deformation and displacement by rounded part of the cutting edge (BC in Fig.1). This is the plastic deformation zone where no chip is formed. In addition to ploughing, particle fracture and displacement also takes place in this region. The particle fracture and displacement is considered to occur mainly along the cutting line (CD in Fig.1). The quantitative estimation of force components due to different processes discussed above are now considered.

\subsection{Chip formation}

As noted earlier, there are similarities in the chip formation mechanism of MMCs to that of monolithic materials such as aluminium or steel. It should be noted that, during machining, shearing occurs in a zone rather than on a plane. However, at relatively higher cutting speeds, the thickness of the shear zone reduces and hence it can be approximated by a shear plane [25]. Due to the simplicity of the shear plane models and relatively high cutting speeds normally used when machining MMCs with PCD tools, Merchant's analysis [31] ${ }^{1}$ was selected for the present investigation to determine the chip formation forces. In Merchant's analysis, the chip is considered as a separate body in equilibrium under the action of two equal, opposing forces: the force which the tool exerts on the back surface of the chip and that the work piece exerts on the base of the chip at the shear plane (AB in Fig.1). Thus the force components acting on the tool in the direction of cutting, $F_{\mathrm{cc}}$, and that in the direction of feed (thrust), $F_{t c}$, can be determined using the equations given below [31]:

\footnotetext{
${ }^{1}$ The theory is limited to orthogonal cutting with plane face tool having single straight cutting edge.
} 


$$
\begin{aligned}
& F_{c c}=\tau_{s} A_{c} \frac{\operatorname{Cos}(\beta-\gamma)}{\operatorname{Sin} \phi \operatorname{Cos}(\phi+\beta-\gamma)} \\
& F_{t c}=\tau_{s} A_{c} \frac{\operatorname{Sin}(\beta-\gamma)}{\operatorname{Sin} \phi \operatorname{Cos}(\phi+\beta-\gamma)}
\end{aligned}
$$

where $A_{c}$ is the cross-sectional area of cut, $\tau_{s}$ is the shear strength of MMC, $\beta$ is the mean friction angle, $\gamma$ is the tool rake angle and $\phi$ is the shear angle which is given by

$$
\tan \phi=\frac{r_{c} \cos \gamma}{1-r_{c} \sin \gamma}
$$

where $r_{c}$ is chip thickness ratio (given by cut-thickness divided by chip thickness). The shear angle, $\phi$, and mean friction angle, $\beta$, are shown to depend on machining conditions, work piece material, etc.

\subsection{Nose radius effect}

Since the PCD tools have nose radii, their influence was taken into account using the concept of equivalent cutting edge. With this method, the straight and round parts of the cutting edge are replaced by a single straight cutting edge, i.e., the equivalent cutting edge which was taken as the line joining the extreme points of the engaged cutting edge as suggested by Colwell [32]. The chip flow over the rake face of the tool was assumed to be perpendicular to the equivalent cutting edge. For the case as in Fig. 3a when $\kappa_{r}=90^{\circ}$ and $a$ is greater than the tool nose radius, the approach angle for the equivalent cutting edge, $\kappa_{r}^{*}$ is given by

$$
\kappa_{r}^{*}=\cot ^{-1}\left(\frac{r_{\varepsilon}+\frac{f}{2}}{a}\right)
$$

and when $\kappa_{r}=90^{\circ}$ and $a$ is smaller than nose radius (Fig. 3(b)), $\kappa_{r}^{*}$ is

$$
\kappa_{r}^{*}=\cot ^{-1}\left\{\frac{\left(2 r_{\varepsilon} a-a^{2}\right)^{1 / 2}+\frac{f}{2}}{a}\right\}
$$

Note that cut thickness $t$ and width of cut $w$ needed in the calculations are determined from $f$ and $a$ in terms of $\kappa_{r}^{*}$, i.e., $t=a / \sin \kappa_{r}^{*}$ and $w=f \sin \kappa_{r}^{*}$. 


\subsection{Ploughing}

Since the particle fracture is considered separately in the present work, only the matrix metal is assumed to be subjected to ploughing. In order to determine the force components due to metal matrix ploughing, the equations given in reference [33] which are based on a slip line field model for a rigid wedge sliding on a half-space and for orthogonal cutting can be used.

With the above model, the ploughing force components acting on the tool in the direction of cutting, $F_{c p}$, and that in the direction of feed, $F_{t p}$, can be determined as

$$
\begin{aligned}
& F_{c p}=\tau_{s m} l r_{n} \tan \left(\frac{\pi}{4}+\frac{\gamma}{2}\right) \\
& F_{t p}=\tau_{s m} l r_{n}\left[1+\frac{\pi}{2}\right] \tan \left(\frac{\pi}{4}+\frac{\gamma}{2}\right)
\end{aligned}
$$

where $l$ is the active cutting edge length, $r_{n}$ is the cutting edge radius and $\tau_{s m}$ is the shear strength of the matrix material. Noting that the width of cut is the active cutting edge length which encompasses straight and round parts of the cutting edge involved in cutting, $l$ is given by

$l=r_{\varepsilon}\left[\kappa_{r}+\sin ^{-1}\left(\frac{f}{2 r_{\varepsilon}}\right)\right]+\frac{a-r_{\varepsilon}\left[1-\cos \left(\kappa_{r}\right)\right]}{\sin \left(\kappa_{r}\right)}$

where $r_{\varepsilon}$ is the tool nose radius, $\kappa_{r}$ is the approach angle, $f$ is the feed and $a$ is the depth of cut.

\subsection{Particle fracture and displacement}

While the tool moves in the direction of cutting, the hard particles in the ploughing zone are fractured or displaced. Thus the cutting edge of the tool can be considered to be responsible for particle fracture/displacement which, therefore, occurs along the cutting edge of the tool. The total energy for particle fracture during orthogonal cutting of MMC can be calculated as

$$
E_{f t}=\mu_{g} l
$$

where $\mu_{g}$ is the average fracture energy per unit cutting edge length in orthogonal cutting. If the force in the cutting direction due to particle fracture and displacement is $F_{c f}$, then $F_{c f} L=E_{f t}=\mu_{g} l$, where $L$ is the cutting distance. $F_{c f}$ is then determined by

$$
F_{c f}=\frac{\mu_{g} l}{L}
$$


In order to determine the force due to particle fracture/displacement in the thrust direction, the direction of the resultant force must be determined first. It is assumed that the reinforcement particles are uniformly distributed and spherical with uniform average diameter, and that the interaction of particles with the cutting edge can be represented by a particle placed at the middle of the ploughing zone as shown in Fig.2. Neglecting the frictional effects during the interaction, the direction of resultant force is determined from the relation

$\sin \delta=\frac{H}{d_{p}+2 r_{n}}$

where $\delta$ is the angle of resultant force measured from the cutting direction, $d_{p}$ is the average diameter of particles and $H$ is the width of the ploughing zone which is given by

$H=r_{n}(1+\sin \gamma)$

Then the component of the force due to particle fracture and displacement acting in the thrust direction is

$F_{t f}=F_{c f} \tan \delta$

Forces $F_{c f}$ and $F_{t f}$ can be determined from Eqs. (8)-(11) when $\mu_{\mathrm{g}}$ is known. The method used to obtain $\mu_{\mathrm{g}}$ will be discussed in Section 4.2.

\subsection{Cutting and thrust forces}

The total forces acting on the tool in the cutting and thrust directions $F_{c}$ and $F_{t}$ are therefore given by

$$
\left.\begin{array}{rl}
F_{c} & =F_{c c}+F_{c p}+F_{c f} \\
F_{t} & =F_{t c}+F_{t p}+F_{t f}
\end{array}\right\}
$$

\section{Experimental procedure}

The experiments were made on a lathe using a bar turning process under dry conditions. For each test condition, it was necessary to measure the cutting forces and chip thickness. These process parameters are affected by the MMC work material (volume/weight percentage of ceramic particles, particle size, heat treatment and aluminium alloy composition, etc), cutting conditions, tool material and geometry, 
etc. Because of the cost of MMC work materials and PCD tools, and to keep the experiments to a manageable number, selected ranges of each parameter are as follows.

(a) MMC work material

It had 20 vol\% SiC particles with particle size $6-18 \mu \mathrm{m}$ in 6061 aluminium matrix (designated as F3S.20S in Alcan's literature). The material was first direct chill (DC) cast and then hot-extruded. The diameter and length of the bar were $90 \mathrm{~mm}$ and $535 \mathrm{~mm}$ respectively.

(b) Tool material and geometry

Polycrystalline diamond (CTH025 grade from Element-6) tipped TPMN 160304 inserts were used on tool holder CTGPR2525-M16. The nose radius was $0.4 \mathrm{~mm}$ while rake and approach angles were $5^{\circ}$ and $90^{\circ}$ respectively. The cutting edge (without edge hone) radius was measured to be $5.42 \mu \mathrm{m}$.

(c) Cutting conditions

The ranges of cutting conditions selected were based on literature and tool manufacture's recommendations. Accordingly, the selected values were: depth of cut 0.25, 0.5, 1.0 and $1.5 \mathrm{~mm}$; feed 0.1, $0.15,0.2$ and $0.25 \mathrm{~mm} / \mathrm{rev}$; cutting speed 100, 200, 400, 600 and $800 \mathrm{~m} / \mathrm{min}$. During experiments, only one of the above parameters was varied while others were held constant to observe the effects of individual parameter on machining forces.

A multi-axis, Mori-Seiki MT2000a1sz CNC turning centre was used for the machining tests. In measuring the cutting forces, a Kistler type 9121 three axis piezo-electric dynamometer was used with data acquisition system that consisted of Kistler multi-channel charge amplifier (type 5019), data taker (DT800) and PII personal computer with data logger software. Before starting the experiments, the three axes of the dynamometer were calibrated using static loads.

For each test condition, cutting was performed for over 10 seconds. The software allowed force signals to be monitored on-line and stored in data taker memory. At the end of tests, all the signals were uploaded to the personal computer for subsequent analysis. A number of replication tests were also made to assess the experimental scatter of the measured parameters. During each test, chip samples were collected for measuring chip thickness. 
Using uploaded data and Microsoft excel package, average force for each test condition was determined. Chip thickness values were measured using a micrometer.

\section{Results and Discussion}

\subsection{Effect of cutting speed}

Cutting speed and feed influence the strength of the work piece material in the deformation zones through temperature, strain and strain rate. In the present work, for simplicity, the effects of strain, strain rate and temperature on shear strength are not considered explicitly. However, it was found that, the measured $F_{c c}, F_{c t}$ and $\phi$ depend on cutting speed. Hence, the experimental shear strength values, $\tau_{s}$, determined by

$\tau_{s}=\frac{\left[\left(F_{c c} \cos \phi\right)-\left(F_{t c} \sin \phi\right)\right] \sin \phi}{A_{c}}$

are also cutting speed dependent. Therefore, if a relationship between $\tau_{s}$ and $v$ can be determined, the effect of the cutting speed can be implicitly accounted for in the model developed in Section 3. As a first approximation, a simple linear relation between $\tau_{s}$ and $v$ was determined using a small number of experimental results as follows.

$\tau_{s}=201.82-0.0102 v$

Additionally, the experimental values of $\phi$ (determined by Eq. (2) ${ }^{2}$ ) and friction angle $\beta$ (determined by an iterative procedure using Eq.(1)) were also found to depend on $v$. Once again, using a small number of experimental results, linear relations for $\phi$ and $\beta$ were developed as follows

$\phi=20.15+0.0072 v$

$\beta=39.73-0.0128 v$

Hence, for a given cutting speed, $\tau_{s}, \phi$ and $\beta$ for determining the chip formation forces can be obtained using Eqs. (14), (15) and (16), respectively.

\footnotetext{
${ }^{2}$ It is interesting to note that, at varying cutting speeds, the relationship between $\phi$ and $(\beta-\gamma)$ observed for monolithic materials [36,37], i.e., $\phi=B-C(\beta-\gamma)$, still approximately holds, even for the cutting of MMCs. A detailed analysis is given in the Appendix.
} 


\subsection{Determination of $\mu_{g}$}

In order to determine the forces due to particle fracture and displacement using Eqs. (8)-(11), the value of $\mu_{\mathrm{g}}$ is required and this must be determined from experiment.

As noted earlier, Yan and Zhang [30] calculated the specific energy for particle fracture during scratching of aluminium alloy based MMCs. They applied Griffith’s theory ${ }^{3}$ to estimate the energy for particle fracture under a pyramid indenter, as shown in Fig. 4. They also determined the specific energy for particle fracture by subtracting the scratching energy of the corresponding aluminium matrix material from the total specific energy of the composites reinforced with $\mathrm{SiC}(10$ and $20 \mathrm{wt} \%)$ and $\mathrm{Al}_{2} \mathrm{O}_{3}(10$ and 20 wt \%) particles. Their results can be used to determine $\mu_{\mathrm{g}}$ because in deriving Eq. (8), we have considered that the tool edge is responsible for particle fracture.

For a given groove depth $h$ (Fig. 4), the specific particle fracture energy $\mu_{\mathrm{f}}$ can be obtained from the results given in reference [30]. The total energy of particle fracture $E_{f}$ for scratching is

$$
E_{f}=\mu_{f} A L=\mu_{f} h^{2} \tan \theta L
$$

where $A$ is the cross-sectional area of groove, $\theta$ is the indenter apex angle between opposite faces and $L$ is the length of scratch. Note that the volume of material removed is $A L=h^{2} \tan \theta L$. The effective indenter edge length $L_{\mathrm{ei}}$ causing particle fracture is given by

$$
L_{e i}=\frac{2 h}{\cos ^{2} \theta}
$$

Hence, the energy of particle fracture per unit cutting edge length $L_{\mathrm{ei}}$ is

$\mu_{g}=\frac{E_{f}}{L_{e i}}=\frac{\mu_{f} h \sin (2 \theta) L}{4}$

The value of $\mu_{g}$ was interpolated from a range of data available in [30], the obtained value of $\mu_{g}=$ $0.01 \mathrm{~J} / \mathrm{mm}$ for $\mathrm{SiC}$ (20 vol \%). Using the given data and Eqs. (8)-(11), the cutting and thrust force components due to particle fracture and displacement are determined for different cutting conditions.

\footnotetext{
${ }^{3}$ According to this theory, the energy required to propagate a crack is equal to the surface energy of the crack surface created.
} 


\subsection{Comparison of predicted and experimental results}

For the conditions used in the tests discussed in Section 3, the predicted forces for chip formation from Eqs.(1) and (2), ploughing from Eqs.(5) and (6) and, particle fracture and displacement from Eqs.(8) and (11) were first determined. These forces were then added up to determine the total forces in the cutting and thrust directions which were then compared with experimental forces ${ }^{4}$ obtained under identical conditions.

Fig.5 compares the predicted and experimental forces with varying feed. In this figure, the experimental force results are represented by symbols and predicted results by lines. As expected, the experimental cutting and thrust forces can be seen to increase more or less linearly with the increase in feed. The rate of increase in cutting force is higher than that of the thrust force. The predicted results show the same trend as the experimental ones. Additionally, excellent quantitative agreement between predicted and experimental results can be seen.

The comparison between predicted and experimental force results with varying depths of cut is presented in the Fig.6. As expected, the experimental and predicted force results also increase more or less linearly with the increase in depth of cut. Once again, a higher rate of increase in cutting force than thrust force as well as excellent qualitative and quantitative agreement between predicted and experimental results is obtained.

The comparison between predicted and experimental force results for varying cutting speed is presented in Fig. 7. Unlike the variations of forces with feed and depth discussed above, in this case, forces can be seen to decrease approximately linearly with increase in speed with somewhat similar rates of decrease. Once again, excellent qualitative and quantitative agreement between predicted and experimental results seems to exist.

In addition to the above comparison between predictions and experimental results obtained from the authors' tests, an attempt is now made to further verify the predictive method by comparing the predictions with the experimental results published in the literature. It was found that only few experimental force results were available. Notably, Davim et al [3], Chambers [7] and El-Gallab et al

\footnotetext{
${ }^{4}$ In line with the equivalent cutting edge concept used in predicting the forces, the experimental thrust force was taken as the resultant force of experimental feed and radial forces.
} 
[12] measured the forces in machining MMCs with PCD tools for a range of cutting speeds. However, none of these papers give data in sufficient detail for comparison. The parameters used or applicable for the studies in $[3,7,12]$ that will be used for further verifying the present model are listed in Table 1. Note that some of the parameters, which were not given in the above papers, have been assumed in accordance with general machining practice or taken from the authors’ investigation.

For the experimental conditions of Davim et al, Chambers and El-Gallab et al, all the components of cutting and thrust forces for different mechanisms were calculated and added according to the procedure described in the forgoing sections.

Fig.8 compares the predicted cutting and thrust force results with experimental results obtained from $[3,7,12]$. The number on abscissa shows the force component and reference from which experimental forces were obtained. The model predictions are shown by bars while the experimental measurements are denoted by circles with variation ranges. It can be seen that the agreement between the predicted and experimental cutting/thrust forces is very good for the conditions used by Davim et al [3] and Chambers [7]. However, the model seems to have largely overestimated the cutting force for the conditions used by El-Gallab et al [12]. This is most surprising when one considers the excellent agreement seen between experimental and predicted results for authors' experimental conditions and those in [3, 7]. It is likely that there is an error either in the equipment used by El-Gallab et al [12] or in the experimental conditions or force results given in [12]. The attempts made to confirm this with the authors of [12] were not successful so far.

Considering the simplifications made in developing the described model, the agreement between predicted and experimental results is very encouraging. This also indicates that chip formation, ploughing and, particle fracture and displacement are indeed the main factors which contribute to the cutting force generation.

\subsection{Comparison with the previous model}

It will be recalled that another theoretical model which, only predicts cutting force, was developed based on energy consumption due to particle debonding and plastic deformation at primary and secondary deformation zone by Kishawy et al [24] as mentioned in the introduction. The predictive 
capability of the present model was compared with that described in Ref.[24]. Predictions made from the energy based model together with those from the present model are given in Fig.5 to 7, which is for cutting conditions typical of those used in the present experiments. From these figures it can be concluded that the energy based model [24] under-predicted the cutting forces at lower feeds but the prediction of that is very well at higher feed. When depth of cut was varied, the energy based model over-estimated the forces at lower depth of cuts and under estimated the forces at higher depth of cuts. The prediction of energy based model is not influenced by cutting speed; hence a horizontal line for cutting forces was obtained with the variation of speed. It is clear from Fig.7 that the predicted line under-estimated the cutting forces at lower speeds and over-estimated the cutting forces at higher speed. From the Figs.5-7 and above discussions it is well proved that the present model is much better in every aspects of machining conditions than that described in Ref.[24] and predicting both the forces very accurately instead of only cutting force by energy based model.

The energy based model consists of three parts such as (i) specific energy due to plastic deformation in the primary deformation zone, (ii) specific energy due to plastic deformation in the secondary deformation zone and (iii) specific energy consumed for debonding the particle from matrix. On the other hand the present model considers (i) chip formation, (ii) ploughing and (ii) particle fracture and displacement for orthogonal cutting. Considering the above facts it can be noticed that the specific energy due to plastic deformation in the primary and secondary deformation zone of energy based model is equivalent to chip formation of the present model. In the same way the particle fracture related part is equivalent to each other for the both models. It seems that the main cause of under prediction of forces at lower feeds by energy based model is due to ignoring ploughing force which plays very important roles at low undeformed chip thickness.

There might be other reasons that influence the predictions of the energy based model negatively. Firstly, the assumption that specific energy due to plastic deformation in the secondary deformation zone is one third of the specific energy due to plastic deformation in the primary deformation zone may not be valid for every machining condition and workpiece materials. The total specific energy for material removal is not constant for all machining conditions and the ratio of energy consumption between 
primary and secondary deformation zone decreases with increase of speed and increases with increase of depth of cut [38]. Hence, at lower depth of cut, energy based model considered higher deformation energy in secondary deformation zone and lower at higher depth of cut, which resulted poor convergences of predicted results with that of experimental. The same is relevant for the poor prediction of forces due to change of speed.

Secondly, for calculating energy due to fracture, Kishawy et al [24] considered only properties of matrix material and reinforcements which may make the poor prediction. Energy for particle fracture and displacement depends on material properties as well as tool geometry and machining conditions [30].

\section{Conclusions}

In this work, a mechanics model was developed for predicting the forces when machining aluminium alloy based MMCs reinforced with ceramic particles. The resultant cutting force was considered to consist of components due to chip formation, ploughing and, particle fracture and displacement, and the calculations of these force components were based on Merchant's shear plane analysis, slip line field theory and Griffith theory respectively. The predictions revealed that, the force due to chip formation is much higher than those due to ploughing and particle fracture. A comparison between predicted and experimental force results showed excellent agreement. Present model was also compared with previous model and superiority of the present model in prediction of cutting and thrust forces was proved.

\section{Acknowledgements}

The authors wish to thank the Australian Research Council and Ringwood Superabrasives Pty Ltd for financial assistance. A. P. has been supported by IPRS and IPA. The authors appreciate Prof. J. P. Davim for providing information not included in his paper.

\section{Appendix}


Here the authors would like to report an interesting phenomenon, i.e., at varying cutting speeds, the relationship between $\phi$ and $(\beta-\gamma)$ observed in machining of monolithic metals [36,37], i.e., $\phi=B-C(\beta$ -

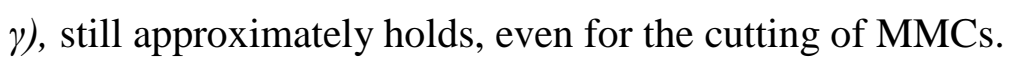

In modeling of chip formation, whether it is shear plane analysis (eg. by Merchant [31], Lee and Shaffer [34]) or shear zone analysis (eg. by Oxley [35]), one of the most important parameters is the shear angle $\phi$, which determines geometry of chip formation and hence cutting forces. The theoretical relations obtained for $\phi$ by Merchant [31] and Lee and Shaffer [34] are:

$$
\phi= \begin{cases}\frac{\pi}{4}-\frac{1}{2}(\beta-\gamma) & \text { Merchant } \\ \frac{\pi}{4}-(\beta-\gamma) & \text { Lee and Shaffer }\end{cases}
$$

However, the experimental results obtained by investigators such as Kobayashi and Thomson [36] and Pugh [37] for a wide range of (monolithic) work materials and cutting conditions show that the following relation is more appropriate for $\phi$.

$\phi=B-C(\beta-\gamma)$

where $B$ and $C$ are constants which depend on the work material.

Fig. 9 shows the experimental values of $\phi$ plotted against $(\beta-\gamma)$ for all the experimental conditions used in the present investigation discussed in Section 3. The linear regression line for the data is also shown in the figure. It can be seen that the experimental results fall close to the line represented by the equation

$\phi=\frac{\pi}{5}-\frac{1}{2}(\beta-\gamma)$

It can be seen that, similar to the case of cutting monolithic materials discussed above, there also exist a linear relationship between $\phi$ and $(\beta-\gamma)$ even in cutting MMCs at varying cutting speeds, although the values of $B$ and $C$ in the case of MMCs (Eq. (21)) are not identical to those of Eq. (20) for monolithic metals.

\section{References}


[1] C. A. C. Antonio, J. P. Davim, “Optimal cutting conditions in turning of particulate metal matrix composites based on experimental and a genetic search model”, Composites: Part A 33(2002) 213219.

[2] Z. F. Zhang, L.C. Zhang, Y. W. Mai, "Wear of ceramic particle-reinforced metal-matrix composites: Part I Wear mechanism”, Journal of Material Science 30(1995) 1961-1966.

[3] J. P. Davim, A. M. Baptista, "Relationship between cutting force and PCD cutting tool wear in machining silicon carbide reinforced aluminium”, Journal of Material processing Technology, 103 (2000) 417-423.

[4] J. P. Davim, "Diamond tool performance in machining metal-matrix composites", Journal of material processing Technology, 128 (2002) 100-105.

[5] N. Narutaki, “Machining of MMC`s”, VDI BERICHTE NR., 1276 (1996) 359-370.

[6] S. Durante, G. Rutelli, F. Rabezzana, "Aluminum-based MMC machining with diamond-coated cutting tools”, Surface and Coating Technology, 94-95 (1997) 632-640.

[7] A. R. Chambers, “The machinability of light alloy MMCs”, Composites: Part A, 27 (1996) 143-147.

[8] R. Polini, F. Casadei, P. D’Antonio and E. Traversa, “Dry turning of alumina aluminium composites with CVD diamond coated Co-cemented tungsten carbide tools”, Surface and coatings Technology, 166 (2003) 127-134.

[9] G. E. D’Errico, R. Calzavarini, “Turning of metal matrix composites”, Journal of Materials Processing Technology, 119 (2001) 257-260.

[10] P. J. Health, “Developments in applications of PCD tooling”, Journal of Materials Processing Technology, 116 (2001) 31-38.

[11] B. Zhao, C. S. Liu, X. S. Zhu, K. W. Xu, "Research on the vibration cutting performance of particle reinforced metal matrix composites SiCp/Al”, Journal of Materials Processing Technology, 129 (2002) 380-384.

[12] M. El-Gallab, M. Sklad, "Machining of Al:SiC particulate metal-matrix composites Part I: Tool performance”, Journal of materials Processing Technology, 83 (1998) 151-158. 
[13] J. P. Davim, "Design of optimization of cutting parameters for turning metal matrix composites based on the orthogonal arrays”, Journal of Materials Processing Technology, 132 (2003) 340-344.

[14] R. M. Hooper, J. L. Henshall, A. Klopfer, "The wear of polycrystalline diamond tools used in the cutting of metal matrix composites”, International Journal of Refractory Metals and Hard Materials, 17 (1999) 103-109.

[15] C. J. E. Andrews, H. Y. Feng, W. M. Lau, "Machining of an aluminum/SiC composite using diamond inserts”, Journal of materials Processing Technology, 102 (2000) 25-29.

[16] G. E. D’Errico, R. Calzavarini, “Turning of metal matrix composites”, Journal of Materials Processing Technology, 119 (2001) 257-260.

[17] P. L. Tso, Y. G. Liu, “ Study on PCD machining”, International Journal of Machine Tools and Manufacture, 42 (2002) 331-334.

[18] F. Nabhani, "Wear mechanism of ultra-hard cutting tools materials”, Journal of Materials processing technology, 115 (2001) 402-412.

[19] N. Tomac, K. Tonnessen, “Machinability of particulate aluminum matrix composites”, Annals of the CIRP, vol. 41/1/1992, 55-58.

[20] L. Cronjager, D. meister, “Machinability of fiber and particle-reinforced aluminum”, Annals of the CIRP, vol.41/1/92, 63-66.

[21] J. T. Lin, D. Bhattacharyya, C. Lane, "Case study-machinability of a silicon carbide reinforced aluminium metal matrix composite”, Wear, 181-183 (1995) 883-888.

[22] A. Manna, B. Bhattacharayya, “A study on machinability of Al/SiC-MMC”, Journal of Materials Processing Technology, 140 (2003) 711-716.

[23] K. C. Chan, C. F. Cheung, M. V. Ramesh, W. B. Lee, S. To, “A theoretical and experimental investigation of surface generation in diamond turning of an Al6061/SiCp metal matrix composite”, International Journal of Mechanical Sciences, 43 (2001) 2047-2068.

[24] H. A. Kishawy, S. Kannan, M. Balazinski, “An energy based analytical force model for orthogonal cutting of metal matrix composites”, Annals of the CIRP, vol. 53/1/2004, 91-94. 
[25] J. T. Lin, D. Bhattacharyya, W. G. Ferguson, "Chip formation in the machining of SiC-particlereinforced aluminum-matrix composites”, Composites Science and Technology, 58 (1998) 285-291.

[26] M. El-Gallab, M. Sklad, "Machining of Al/SiC particulate metal matrix composites Part II: Work surface integrity”, Journal of Material Processing Technology, 83 (1998) 277-285.

[27] R. Karthikeyan, G. Ganesan, R.S. Nagarazan, B. C. Pai, “A critical study on machining of Al/SiC composites”, Materials and Manufacturing Processes, 16(1) (2001) 47-60.

[28] N. P. Hung, S. H. Yeo, k. K. Lee, K. J. Ng, “Chip formation in machining particle-reinforced metal matrix composites”, Materials and Manufacturing Processes, 13(1) (1998) 85-100.

[29] N. P. Hung, N. L. Loh and V. C. Venkatesh, "Machining of metal matrix composites”, in Machining of ceramics and composites, (Ed: S. Jahanmir, M. Ramulu, P. Koshy), Marcel Dekker Inc., New York, 1999.

[30] C. Yan and L. C. Zhang, "Single-Point Scratching of 6061 Al Alloy reinforced by Different Ceramic Particles”, Applied Composite Materials, 1(1995) 431-447.

[31] M. E. Merchant, “Mechanics of the metal cutting process. I. Orthogonal cutting and type 2 chip”, Journal of Applied Physics, (1944) 267-275.

[32] Colwell, L. V., "Predicting the angle of chip flow for single-point cutting tool”, Transaction of the ASME, 76 (1954) 199-204.

[33] D. J. Waldorf, “A Simplified Model For Ploughing Forces In Turning”, Transaction of NAMRI of SME, 32 (2004) 447-454.

[34] E. H. Lee, B. W. Shaffer, "The theory of plasticity applied to a problem of machining”, Journal of Applied Mechanics, December (1951) 15-20.

[35] P.L.B. Oxley, The mechanics of machining: an analytical approach to assessing machinability, Ellis Horwood, Chichester, 1989.

[36] S. Kobayashi, E. G. Thomsen, "Some observations on shearing process in metal cutting", Transactions of the ASME, Journal of Engineering for Industry, August (1959) 251-261.

[37] H. Ll. D. Pugh, “Mechanics of cutting process”, Proceedings, Conference on Technology of Engineering Manufacture, The Institute of Mechanical Engineers, (1958) 237-254. 
[38] M. C. Shaw, "Metal cutting principles, Oxford Science Publications, Clarendon Press, Oxford, 1989, pp 30-36. 


\section{Captions for figures and tables}

Fig.1. Machining process of MMC.

Fig.2. Interaction of a particle with cutting edge.

Fig.3. The equivalent cutting edge for nose radius tools.

Fig.4. Schematic diagram of a scratched groove [30].

Fig.5. Comparison of predicted and experimental forces with varying feed.

Fig.6. Comparison of predicted and experimental forces with varying depth of cut.

Fig.7. Comparison of predicted and experimental forces with varying cutting speed.

Fig.8. Comparison between predicted and experimentally measured forces from literature

Fig.9. $\phi$ versus $(\beta-\gamma)$ relationship for machining of MMC by PCD tool

Table1. Machining parameters used in the investigations in $[3,7,12]$ 


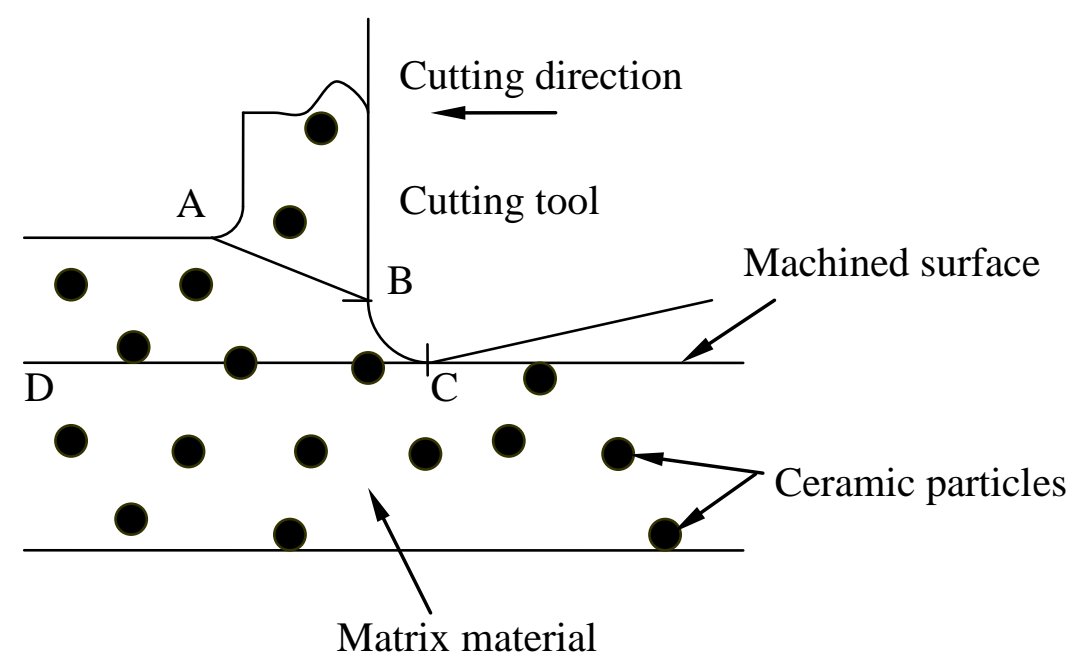

Fig.1

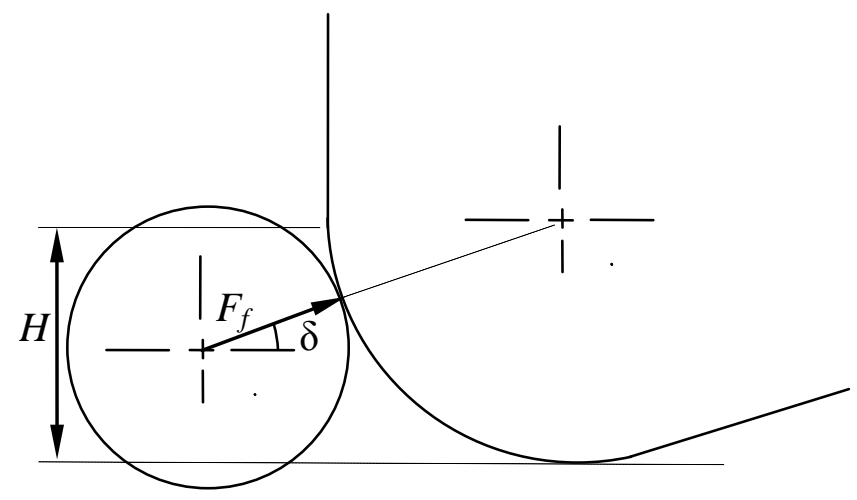

Fig.2

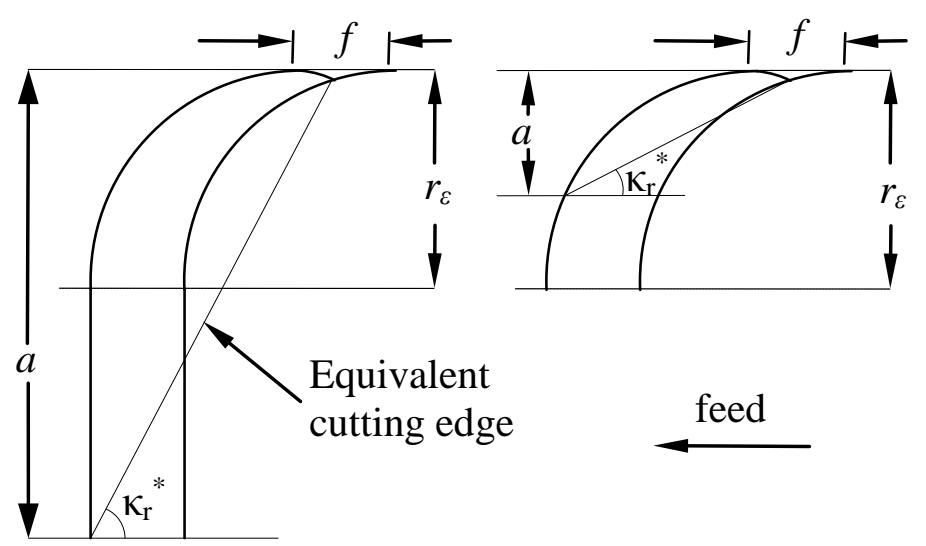
(a) When $r_{\varepsilon}<a$
(b) When $r_{\varepsilon}>a$

Fig 3 


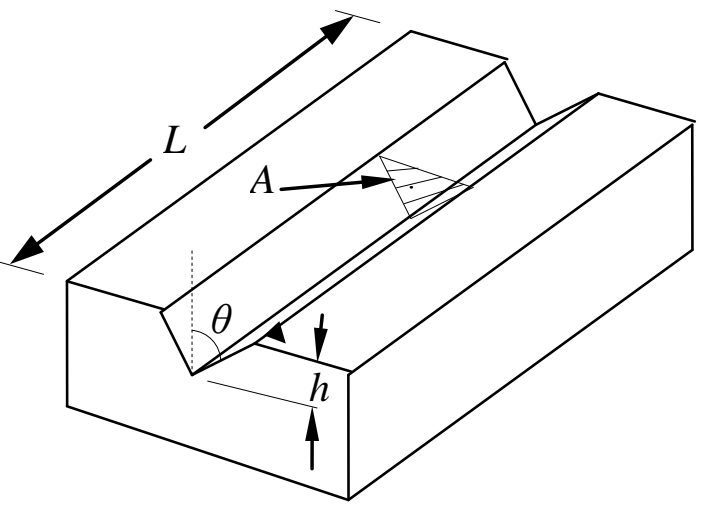

Fig.4

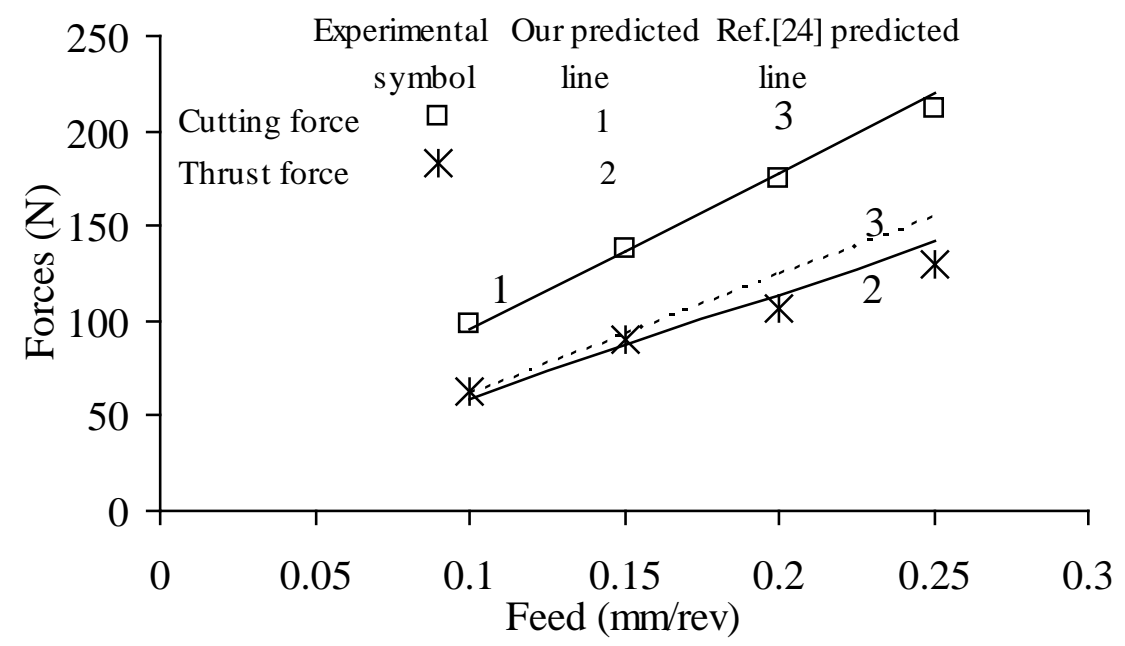

Fig.5

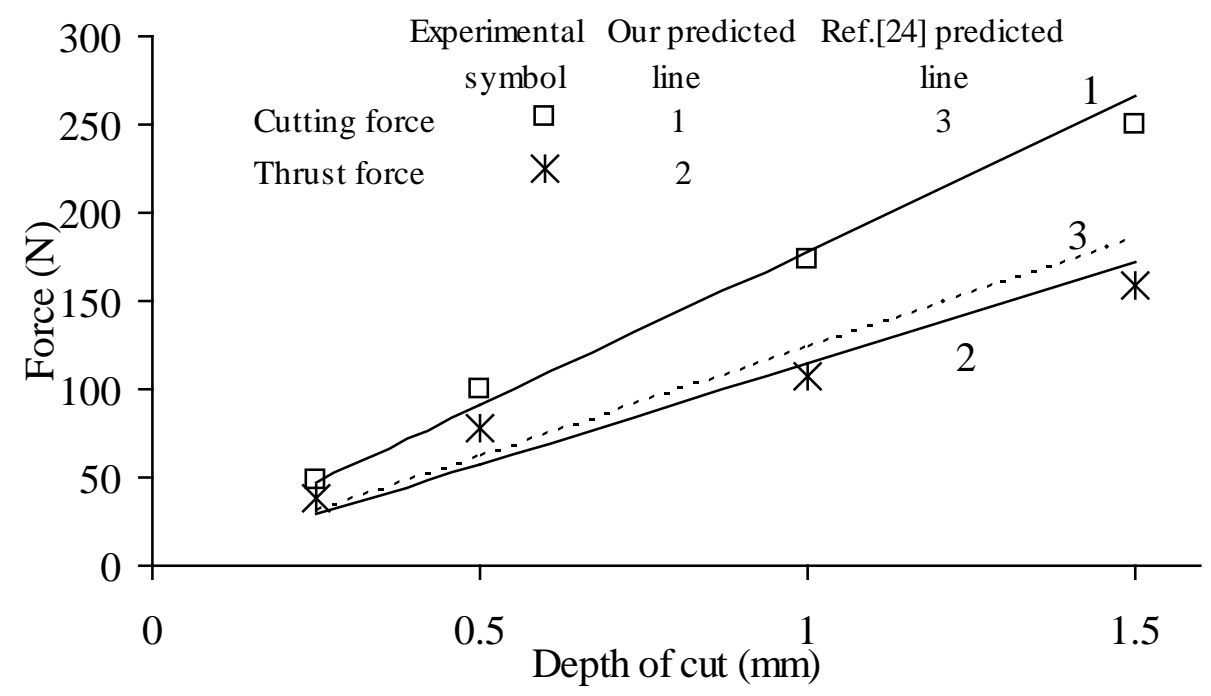

Fig.6 


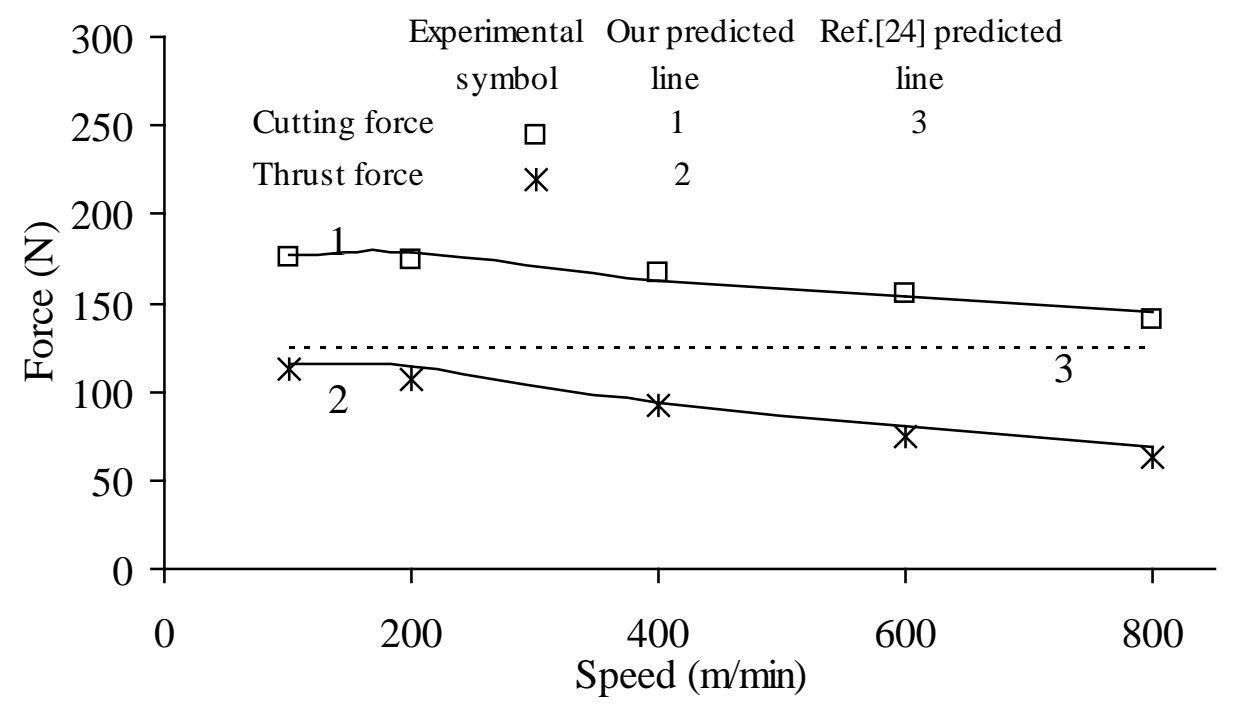

Fig. 7

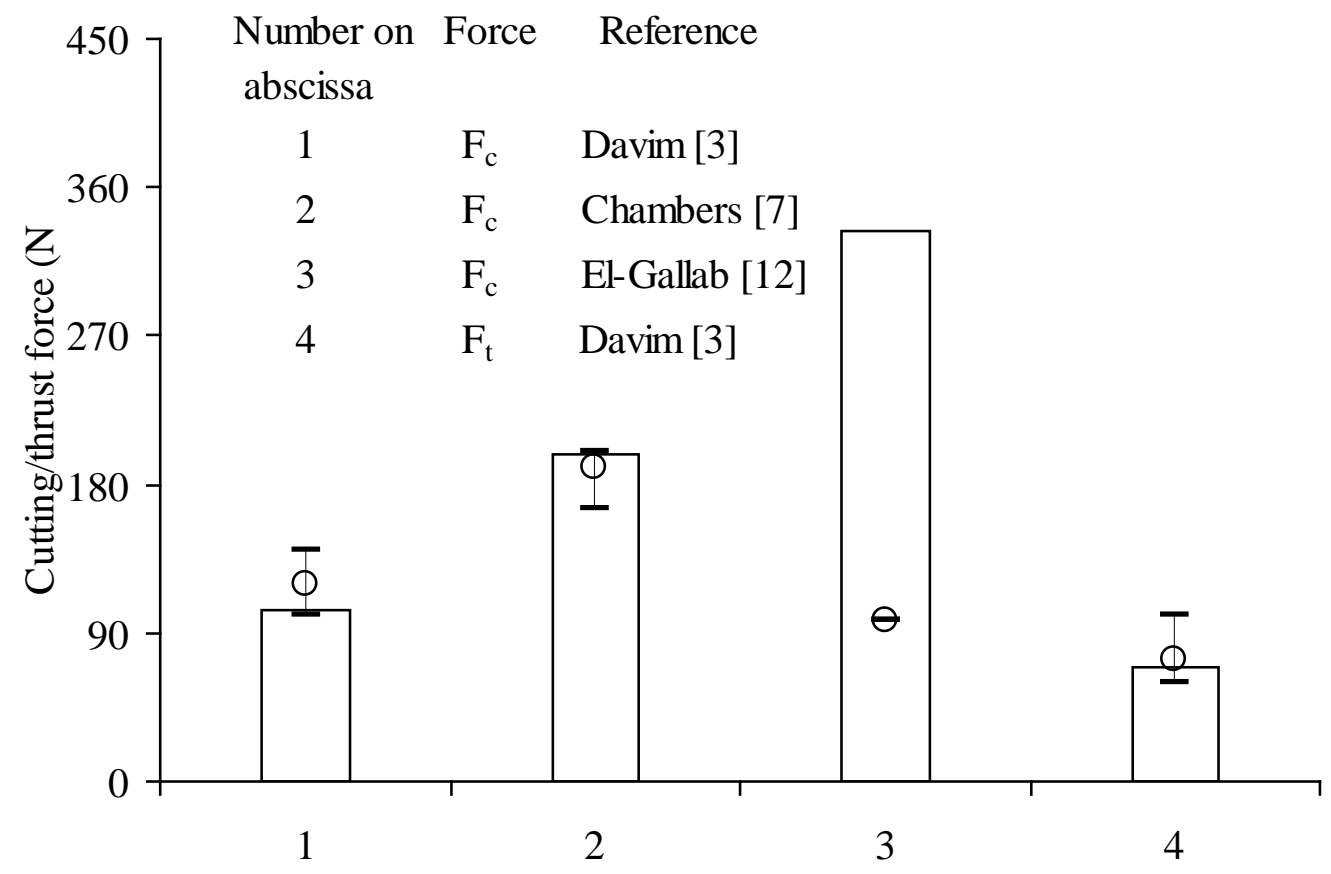

Fig.8 


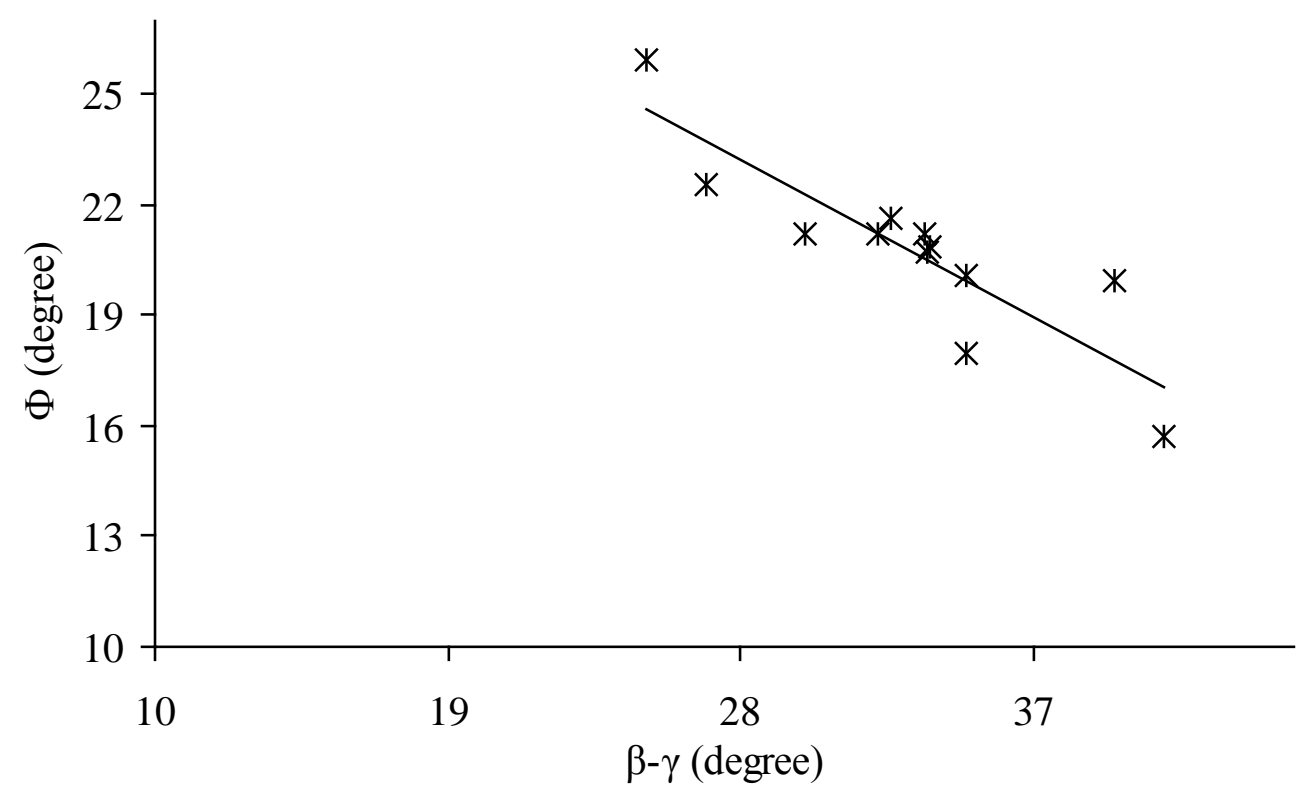

Fig. 9

Table 1

\begin{tabular}{|l|c|c|c|}
\hline \multicolumn{1}{|c|}{ Parameters } & Davim et al[3] & Chambers [7] & El-Gallab et al[12] \\
\hline Tool material & PCD & PCD & PCD \\
\hline Nose radius, $r_{\varepsilon}(\mathrm{mm})$ & 0.8 & 1.6 (assumed) & 1.6 \\
\hline Rake angle, $\gamma($ degree) & 0 & 0 & 0 \\
\hline $\begin{array}{l}\text { Approach angle } \\
\text { (degree) }\end{array}$ & 85 (assumed) & 85 (assumed) & 85 \\
\hline Cutting speeds $(\mathrm{m} / \mathrm{min})$ & $250-700$ & $50-300$ & 670,894 \\
\hline Feed, $f(\mathrm{~mm} / \mathrm{rev})$ & 0.1 & 0.2 & 0.25 \\
\hline Depth of cut, $a$ (mm) & 1 & 1 & 1.5 \\
\hline $\begin{array}{l}\text { Workpiece material } \\
\text { A356-20\%SiC- } \\
\text { Yield strength of matrix }\end{array}$ & 138 & A356-15\%SiC & A356-20\%SiC- T71 \\
$($ MPa $)$ & 20 & 22.5 & 124 \\
\hline $\begin{array}{l}\text { Average particle } \\
\text { diameter, } d_{\mathrm{p}}(\mu \mathrm{m})\end{array}$ & & & 12 \\
\hline
\end{tabular}

